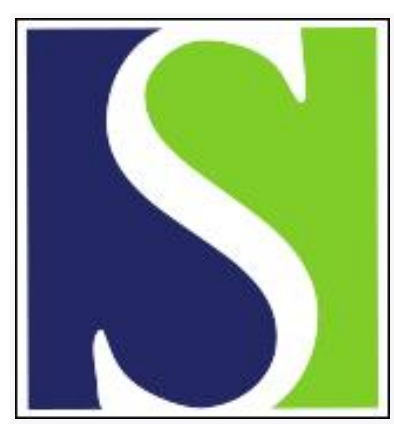

Scand J Work Environ Health 1985;11(3):173-180

https://doi.org/10.5271/sjweh.2237

Issue date: Jun 1985

\title{
Strategies of air sampling.
}

by Corn $\mathrm{M}$

This article in PubMed: www.ncbi.nlm.nih.gov/pubmed/4035319

\section{(c) (i)}




\title{
Strategies of air sampling
}

\author{
by Morton Corn, $\mathrm{PhD}^{1}$
}

\begin{abstract}
CORN M. Strategies of air sampling. Scand J Work Environ Health 11 (1985) 173-180. Considerations relevant to preparation for air sampling are reviewed, including purpose, recognition of need, and the logistics of obtaining the samples (where, when, how, and for how long). Three strategies of sampling focused on how many samples to obtain are discussed in detail, ie, the zoning strategy and strategies of the National Institute for Occupational Safety and Health (NIOSH) and the Mining Safety and Health Administration (MSHA). The purpose of the zoning method is to locate groups of employees with high exposures, while the NIOSH method aims at determining compliance of individual sample results with a regulatory standard. Similarities and differences between the methods are highlighted. The MSHA strategy is completely regulatory and represents a scientifically indefensible strategy. Finally, the advantages of incorporating the assumption of a distribution of workplace contaminant-in-air concentrations are noted.
\end{abstract}

Key terms: chemical hazards, dust, environmental monitoring, occupational hygiene.

The introduction of national legislation for occupational safety and health during the 1970 s and 1980 s was accompanied by prescriptive mandatory standards for potentially toxic chemical and physical agents. The concept of a permissible exposure limit (PEL) is an essential ingredient of standards for toxic agents. Prior to these regulations, industrial hygienists sampled the air for chemical content, but data so obtained were judged to be acceptable or unacceptable on the basis of experience and a general perusal and evaluation of a limited number of sampling results. The hygienist did not have to defend his/her results on the basis of statistical reliability of the data, adherence to standard methods of sampling and analysis, utilization of certified personnel for sampling and certified laboratories for sample analyses. Today all of these considerations are relevant to a program for sampling the air of a worksite, at least in the United States (US), and increasingly in other countries. As indicated elsewhere, the strategy of air sampling is a term reserved for the logistical approach to obtaining a number of samples consistent with the desired confidence in the accuracy and reliability of the measured concentrations (2). However, this statement implies that the questions of where, when, for how long, etc, have also been answered in relation to the sampling of a particular site. Recent personal experience suggests that this assumption is false and that a total strategy of air

\footnotetext{
1 The Johns Hopkins University, School of Hygiene and Public Health, Division of Environmental Health Engineering, Baltimore, Maryland, the United States.
}

Reprint requests to: Dr M Corn, The Johns Hopkins University, School of Hygiene and Public Health, Division of Environmental Health Engineering, 615 North Wolfe Street, Baltimore, MD 21205, USA. sampling is usually not the basis for often extensive collections of air samples.

In addition to the general scientific defensibility of the air sampling program, a carefully constructed strategy of air sampling can optimize the number of samples obtained to capture the desired data and thus reduce the cost of the program. This is a major consideration because the repetitive, cyclic nature of air sampling is usually not understood by private sector line managers who are faced with the often large costs of environmental monitoring. Efficiencies of cost help to "sell" such programs on an ongoing basis.

The purpose of this paper is to review the status of strategy codification for air sampling and to introduce selected new approaches. The relevance of all strategies to regulatory requirements must be considered because compliance with legal standards is an everyday reality for plants operating in many countries. However, the so-called "regulatory" strategy often captures the least information for utilization and should not, in my opinion, be the primary influence on strategy selection. Data needed to demonstrate compliance with the regulatory requirements will often be a spin-off of other sampling strategies.

\section{Preliminary considerations}

\section{Purpose of sampling}

Air sampling can be undertaken for several purposes as, for example, those listed in table 1. Each of the purposes included in table 1 must be carefully considered for the development of an effective air sampling strategy. Considerations relevant to this first phase of strategy development follow. 
Table 1. Selected purposes of air sampling.

\begin{tabular}{|c|c|c|}
\hline $\begin{array}{l}\text { Evaluation of individual employee } \\
\text { exposure }\end{array}$ & $\begin{array}{l}\text { Evaluation of sources of } \\
\text { contaminant in a work area }\end{array}$ & $\begin{array}{l}\text { Evaluation of trends of work- } \\
\text { place air contamination }\end{array}$ \\
\hline $\begin{array}{l}\text { Conformance with a regulated standard } \\
\text { (in the United States an } 8-h \text { permissible } \\
\text { exposure level or short-term ceiling } \\
\text { limit) }\end{array}$ & Process change effects & In terms of employee exposure \\
\hline $\begin{array}{l}\text { Long-term average exposure } \\
\text { (for epidemiologic studies) }\end{array}$ & Process efficiency monitoring & $\begin{array}{l}\text { In terms of selected "air parcels" } \\
\text { at fixed locations }\end{array}$ \\
\hline $\begin{array}{l}\text { Long-term average exposure of group } \\
\text { members with similar exposures }\end{array}$ & $\begin{array}{l}\text { Daily controls for process control } \\
\text { or employee management } \\
\text { (administrative controls) }\end{array}$ & \\
\hline
\end{tabular}

\section{Other considerations}

Recognition of need. First it must be considered whether there is a consensus among professional staff, employees, and managers that air sampling should be undertaken. Obviously, if the contaminant is regulated, the need exists, but in the US only about 500 chemical agents are regulated out of a total of about 67000 chemicals in commercial usage. In the vast majority of cases the need for sampling is not obvious. If there are employee complaints, the presence of odors, or visible emissions, the need for sampling will be stimulated.

Increasingly, the need for sampling must be established through a prioritization of the chemical agents used so that the potentially most hazardous, or those associated with the highest degree of risk, will receive focus. Some of the factors related to degree of risk are (i) number of persons potentially exposed, (ii) inherent toxicity of the material, (iii) quantities used, (iv) anticipated time and concentration of exposure, (v) confidence in engineering controls, (vi) anticipated changes in process or controls, and (vii) presence of other agents which may be toxicologically synergistic or antagonistic.

It is not uncommon to establish air sampling priorities from a list of 1000 or more chemicals in a small to medium-size facility. In a large facility heavily dependent on chemical utilization, thousands of candidate chemicals must be reduced to a feasible number of high-priority candidates for air sampling. With the passage of time, all chemicals of concern will be evaluated, but this process may literally require years, and the establishment of priorities is a very important aspect of a strategy.

US manufacturers are supposed to offer material safety data sheets (MSDS) for their products; these sheets permit users to protect themselves, where necessary. The toxicologic information listed in the sheets is often scant or totally absent, however, and the setting of priorities for sampling can thus be particularly difficult. A major thrust of current research is the prediction of chemical toxicity on the basis of structure/activity relationships.

The recognition of need also involves informing management and employees of the selection of agents for sampling and why they were selected. In this way, a program will be launched in a climate of understanding rather than fear. These caveats apply to a short survey or to a long-term monitoring or surveillance program. Failure to deal adequately with this aspect of the sampling strategy has resulted, for example, in accusations of my performing time/motion studies and of air pumps being suspected of being tape recorders!

Determination of the degree of the problem. A set of "range finding" or probe samples may be obtained to confirm or reject extreme concerns for exposure on the basis of a recognition of need priorities. A preliminary set of samples need not accurately portray exposure. The purpose of the exercise is to confirm or reject concerns of extreme exposure; it is a "ballpark" or order of magnitude study.

Obtainment of the sample. Samples for the assessment of human exposure should be personal samples collected on the individual employee wearing the sampler. Samples for process control or for trend determination need not be personal samples. Samples collected at locations near sources or continuousmonitoring instrumentation opportunely located will fulfill this purpose. Sometimes it is possible to correlate fixed location monitors with personal exposure below some maximum by locating the continuous monitor(s) at the highest concentration point(s). The use of audible or visual alarms alerts employees to the presence of an excursion in the airborne agent concentration.

Samples must be obtained by qualified individuals. Sampling data are recorded onsite. The growth of a class of industrial hygiene technicians is a major feature of the 1970-1980 period. These individuals are not hygienists, but work under the supervision of a hygienist. They are knowledgeable about the technical details of sampling. Finally, if personal samples are to be obtained, the consent of those who will wear samplers should be obtained and recorded.

\section{General sampling strategy}

Preparation for sampling. Preparation for sampling involves an analysis of the available methods for 


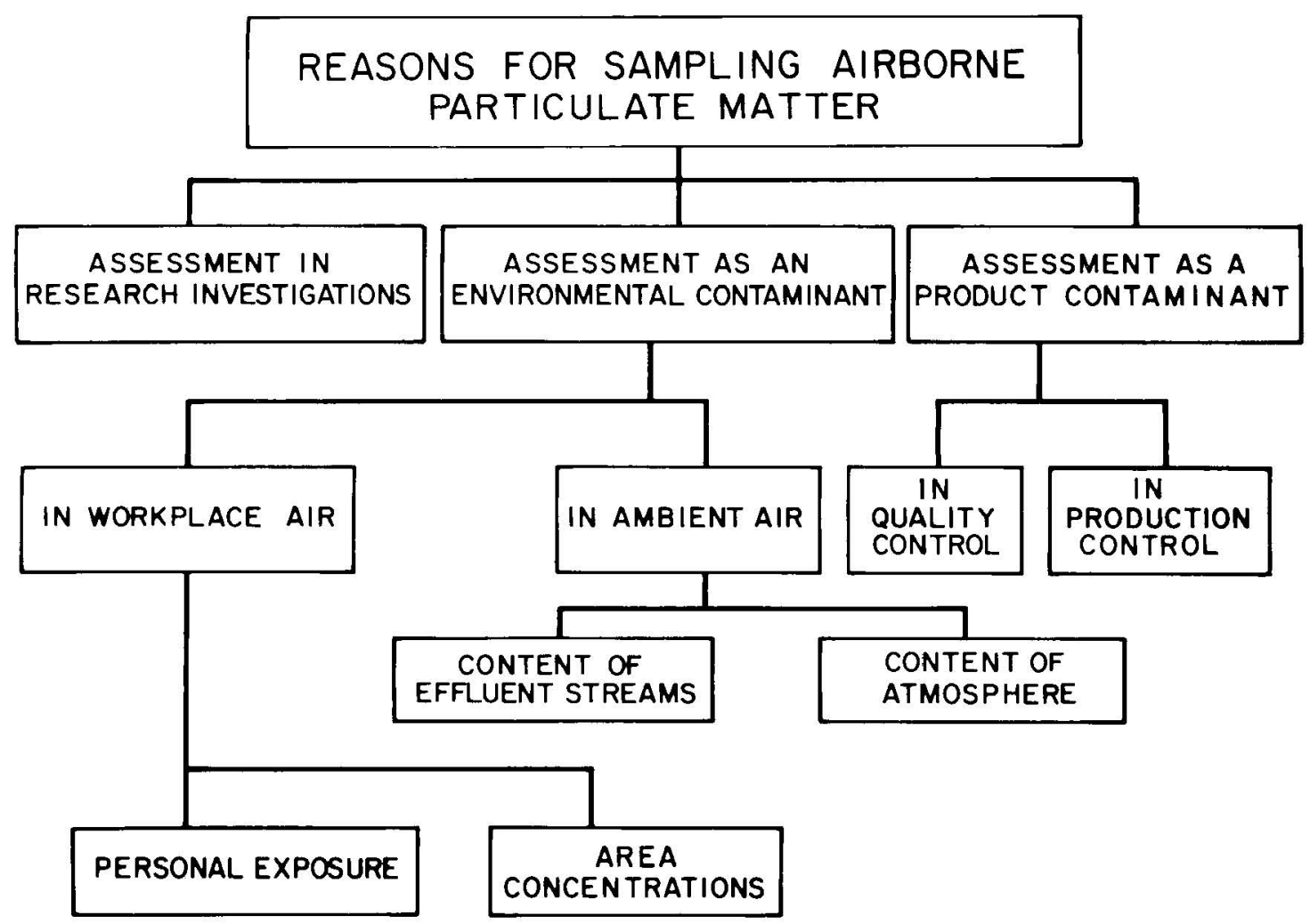

Figure 1. Scheme for the selection of instrumentation for sampling airborne particulate matter (3).

sampling to ensure that the sampling objective is fulfilled. Figure 1 is a previously published schema to address the selection of instrumentation for sampling airborne particulate matter.

Once selected, sampling instrumentation must be calibrated. Calibration referenced to a primary standard should be conducted at least yearly. It is customary to calibrate to a secondary standard prior to and immediately following sampling in the field. In the case of air pumps, it has been common practice to calibrate several times during the course of sampling. A recent development in commercially available pumps has been the self-correcting constant flow pump, which eliminates the need for calibration checks during sampling.

Where to sample. There are suggestions in the literature $(8,10)$ that the "breathing zone," defined as a zone of air of one-foot $(30.5 \mathrm{~cm})$ radius about the head of the sampled individual, is not a homogeneous space. At the present state of the art, samplers can be located anywhere in this zone and adhere to accepted practice. As we learn more about variations in contaminant concentration in the breathing zone, practice may change to a more definitive sampler location.

Sampler placement at a fixed location should be consistent with the sampling objective. If exhaust hood efficacy is being measured, the location should be immediately outside the hood. Regardless of final placement, documentation of the sampler location by three dimensional coordinates is essential. A valid question to ask is, "Could another person precisely locate the sampler in the absence of anyone involved in the current sampling program?',

When to sample. The concentrations of airborne contaminants vary from hour to hour, from day to day, and from season to season. Often there are significant differences in concentrations associated with first, second, and third shifts. A strategy of sampling should incorporate sampling to estimate the full range of concentrations, or, if resources are strictly limited, to target the highest concentrations.

Ideally, sample results should reflect average airborne contaminant concentrations and the degree of variability.

Sampling duration. The first relevant factor is that a sufficient sample be collected for analysis. The anticipated contaminant collection efficiency and the sensitivity of the analytical method will, together, determine the minimum sampling time to meet this requirement. Close coordination with analytical laboratory staff is necessary to meet their needs. It is common practice for many laboratories to supply those taking samples with the sampling cassettes, fil- 
ters, solutions, etc, which are returned to the laboratory after the sampling. These procedures ensure the use of appropriate methods where standard techniques are employed.

The second relevant factor involves matching the sample duration to the anticipated biological effect. Again, where standardized methods are used, these decisions have been made. In the absence of specification, short-term samples (15 min or less) are usually geared to the assessment of irritants, asphyxiants, sensitizers, and allergenic agents. The chronic disease agents are usually sampled for a full shift $(7 \mathrm{~h}$ or more per sample) because short-term fluctuations are not meaningful in the body response. These generalizations are not relevant to the evaluation of collection equipment performance, trends in contaminant concentrations in an area, etc.

Sampling duration and time of sampling become particularly important when biological exposure indices (BEI) are used in conjunction with environmental monitoring. Such indices lag the environmental exposure and often damp exposure peaks. Therefore, careful coordination of measurements in the two programs is essential.

Number of samples. In order to estimate the samples required to obtain the average exposure within a given confidence limit, one must have some idea of the nature of the population distribution and its variance. Most environmental contaminant concentrations adhere to the logarithmic normal distribution (6), a distribution characterized by two parameters, the geometric mean $(\mathrm{Mg})$ and the standard geometric deviation $(\sigma \mathrm{g})$. In the absence of any reduced sampling information, a value of $\sigma \mathrm{g}=2.2$ to 2.5 can be selected to estimate required sample numbers within any desired confidence interval. In any event, a minimum of three samples should be taken before any statement of results is made. If the

Table 2. Suggested monitoring frequency guide.

\begin{tabular}{lc}
\hline Situation & $\begin{array}{c}\text { Sampling } \\
\text { frequency } \\
\text { (months) }\end{array}$ \\
\hline $\begin{array}{l}\text { Control system confidence low } \\
\text { Exposure level not far below the action level }\end{array}$ & 3 \\
$\begin{array}{l}\text { Chemical a serious systemic poison } \\
\text { Odor threshold above the level of the hygienic } \\
\text { standard }\end{array}$ & 3 \\
$\begin{array}{l}\text { Control system confidence fair } \\
\text { Exposure level more than half of the }\end{array}$ & 3 \\
$\begin{array}{l}\text { action level } \\
\text { Odomical a moderate systemic poison }\end{array}$ & 6 \\
level of the hygienic standard & 6 \\
$\begin{array}{l}\text { Control system confidence good } \\
\text { Exposure level less than half of the action level }\end{array}$ & 12 \\
$\begin{array}{l}\text { Chemical a simple upper respiratory irritant } \\
\text { Odor threshold well below the level of the } \\
\text { hygienic standard }\end{array}$ & 12 \\
\hline
\end{tabular}

results of the measurements exceed a $25 \%$ spread, additional samples should be obtained.

When the conclusion is reached that subsequent routine monitoring is necessary, the site, method, and frequency for sampling must be specified. In the absence of a regulatory mandatory schedule or other reasons for departing from in-house considerations of degree of risk, table 2 suggests a monitoring frequency guide.

Record keeping. A minimum record-keeping program involves the following factors for each sample: date, time of sample, location or zone of sampling or person sampled for a personal sample, method of sampling, sample identifier, analytical method, laboratory used, person obtaining sample, and whether or not personal protective equipment was in use at the time of sampling.

Because sampling results rapidly increase paper volume, many organizations use computerized systems to store results. Computerization permits easy data access and analysis and works well, particularly with microcomputers at plant sites.

\section{Selected specific strategies}

Three approaches to a sampling strategy to meet the ongoing needs of a manufacturing facility are reviewed next. Strategies of sampling are also needed for initial surveys and for the start-up of facilities or a process change. But these purposes are served by one-time efforts, and the sampling strategies are not as complex as those considered in this paper.

One method reviewed is known as the zoning method (4). It uses zoning to group employees on the basis of job and environmental similarity prior to sampling. A random selection of employees in each zone is then chosen for personal sampling. The advantage of the method is that for a given number of samples, zoning is more efficient in determining high exposure areas than strict random sampling because it reduces the amount of variation expected in the samples. Attention can then be focused on identified problem areas, and corrective action can be taken.

The second method, described by the US National Institute for Occupational Safety and Health (NIOSH), is a sampling plan to evaluate exposures to airborne chemical contaminants on the basis of statistical theory for deciding compliance or noncompliance with safe exposure limits (7). Employees sampled are those believed to have the highest exposure. Random sampling techniques are only used if maximum risk employees cannot be identified. The measurement of exposures and the evaluation of the results use statistical procedures that consider variations in exposure concentrations caused by the sampling, analysis, and environment. The advantage of the method is it can be applied fairly easily to most industrial hygiene 
sampling, and it is the basis for existing and proposed federal exposure-monitoring regulations.

The third method, referred to as the Mine Safety and Health Administration (MSHA) strategy for evaluating respirable coal mine dust and free silica content of this dust, combines features of the first two methods, but also illustrates certain glaring weaknesses of a major strategy which results in collection of over 200000 samples each year and regulatory action which closes hundreds of mine sections each month (5). Fortunately, this strategy is undergoing review by the US Occupational Safety and Health Administration (OSHA).

\section{Zoning method}

The zoning method of industrial hygiene sampling (4) is used to maximize the effectiveness of sampling to assess risk by zoning workplaces on the basis of similarities of personnel exposure to airborne agents. The following discussion describes how the method is used.

Zone assignment. A zone is defined as a characteristic grouping of workers based on their job similarity and the similarity of the environment in which they work. Each zone must fulfill the following four basic requirements.

1. Work similarity - employees in the zone must perform similar tasks such that similar mechanisms are present for generating air contaminants.

2. Similarity with respect to hazardous agents zone members must use the same agents, and the potential for exposure to the agents under consideration must be similar. Where multiple agents are present, the potential for exposure to the agents must be the same for all persons included in the zone.

3. Environmental similarity - ventilation, process equipment, etc, must be similar for all zone members.

4. "Identifiability" - the same employee must not be classifed in more than one zone. This requirement is important for the random selection of workers from a zone. Employees classified in a zone must qualify for preselection from company records by some means of no-name identification, such as occupational title and department. This process characterizes the work with respect to exposure such that any person with any occupational title identified in a particular zone can be expected to have the exposure value that represents the zone. The procedure of selecting zones involves an examination of all processes, job classifications, material inventories, and ventilation systems in the facility. With this information, and the job classifications by department, the em- ployees can be preliminarily assigned to zones. The employees in each zone are then observed to see if changes in zone assignment need to be made. The plant may be rezoned for subsequent surveys, but the zones selected for a particular survey should not be changed on the basis of the results.

Sampling. After the cohort size in each zone is determined, an appropriate number of workers from each zone is randomly selected for sampling purposes. Procedures for determining the sample size required to ascertain the mean of a population are used on the assumption of a logarithmic normal distribution of measured concentrations and a $\sigma \mathrm{g}$ of 2.3.

Analysis of data. The analysis of data is based on the log-normal distribution of the concentration measurements. The geometric mean, the geometric standard deviation, and the arithmetic mean for each zone and agent combination are calculated. The means are compared to the standard for that agent. If a mean is greater than the standard, at least one of the employees in the zone is exposed to the agent at levels greater than the standard. If the means are less than the standard, the probability of finding employees with exposure in excess of the standard is determined. The expected number of workers with exposure in excess of the standards can also be calculated.

Through data analysis, the zones where employees are expected to have exposure in excess of the standard are identified so that attention can be focused on problem areas and corrective action can be taken. For a given number of industrial hygiene samples, zoning is shown to be more efficient in determining high exposure areas than strict random sampling.

\section{Method described by the National Institute for Occupational Safety and Health}

The NIOSH method of industrial hygiene sampling (7) is a statistically based sampling strategy designed to minimize sampling burden while providing protection to exposed employees by deliberately controlling random measurement error.

Assessment of the possibility of employee exposure. Material inventories, knowledge, and observation of the processes, past sampling data, and information about the physical and chemical characteristics of a material are used to determine whether a toxic or hazardous material in the workplace is released into the workplace air. When the possibility exists, sampling is done to determine whether any employee exposures are in excess of a standard or recommended exposure limit. 
Selection of employees for sampling. The employees believed to have the highest exposures are chosen for personal sampling. The "maximum risk employees" must be identified for every operation and are generally the employees closest to the source of the hazardous material being generated. This may not always be the case in that ventilation patterns, etc, may result in a more remote employee being at maximum risk. Presampling walk-through surveys are required for selecting the maximum risk employees.

If a maximum risk employee cannot be selected for an operation with reasonable certainty, a random selection of employees is made for sampling from the population of workers performing the operation. The subgroup of adequate size is chosen so that there is a high probability that the random sample will contain at least one worker with high exposure, if one exists. Tables are available to help select the adequate subgroup size.

Data analysis. The analysis of data is based on a lognormal distribution of the concentration measurements. The one-sided upper confidence limit is calculated at the $95 \%$ confidence level for each sample. The practical interpretation of a $95 \%$ one-sided upper confidence limit is that one can be $95 \%$ confident that the true average exposure is less than the upper confidence level. Higher levels of confidence can be used for greater degrees of confidence. The confidence limit calculated is compared to the standard or recommended exposure level. If the upper confidence limit is above the standard, a possible exposure above the standard exists. If the upper confidence limit is below the standard, there is $95 \%$ confidence that an employee's exposure is below the standard.

Follow-up sampling. Based on the results of the sampling, a schedule is prepared for further sampling. If the upper confidence limit of the exposure measurement is below the standard, no further sampling is required except if there is a process or control measure change, although sampling of these operations may be put on a periodic basis, such as annually. If the upper confidence limit of the exposure measurement is above the standard, corrective action is taken to lower employee exposure, and the operations are resampled on a monthly or quarterly basis until levels

Table 3. Five-sample designated-area sampling scheme of the Mine Safety and Health Administration - Basis for noncompliance.

\begin{tabular}{cc}
\hline Number of samples & Average concentration $\left(\mathrm{mg} / \mathrm{m}^{3}\right)$ \\
\hline 1 & 10.4 \\
2 & 5.2 \\
3 & 3.5 \\
4 & 2.6 \\
5 & 2.0 \\
\hline
\end{tabular}

are below the standard for two consecutive measurements at least one-week apart.

\section{Enforcement strategy of the Mine Safety and Health Administration}

MSHA utilizes the following strategy to enforce a $2-\mathrm{mg} / \mathrm{m}^{3}$ respirable coal mine dust standard (5). Samples can be of a "designated area" or "designated occupation" type. The "designated area" sample is one collected in the atmosphere of a designated area of the nonface active workings of the mine. It is usually a maximum exposure location based on previous sampling results. "Designated occupation" means the occupation in a mechanized mining unit that has been determined by results of respirable dust samples to have the greatest respirable dust concentration and is considered the occupation most directly affected by the primary dust sources in a mechanized mining unit.

The strategy calls for five designated area samples to be taken during the first month of each bimonthly period. The permissible exposure limit for the samples, as they are submitted by the operator, are shown in table 3. Those familiar with the inaccuracies of respirable dust sampling and concentration determination will appreciate the low margin of error allotted the operator in this sampling scheme. Thus, a single sample of $10.4 \mathrm{mg} / \mathrm{m}^{3}$ curtails the sampling cycle; noncompliance is demonstrated. A violation is issued, and a sequence of regulatory requirements is initiated. Designated occupation sampling proceeds in a similar manner.

The determination of silica content of respirable coal mine dust is the result of a single 8-h sample collected by an MSHA inspector. This procedure triggers calculation of the permissible respirable dust concentration, below $2 \mathrm{mg} / \mathrm{m}^{3}$ if the free silica content exceeds $5 \%$. Once again, known variations in free silica content of respirable coal mine dust, sampling inaccuracies, and chemical analytical procedure inaccuracies make this strategy of sampling scientifically indefensible (5). The result of violation for free silica content of respirable coal mine dust is closure of the section where the sample was obtained. The MSHA strategy is totally regulatory. Despite collection of literally millions of samples, it provides only a limited data base for future epidemiologic studies.

\section{Discussion of strategies}

The zoning and NIOSH sampling plans are similar in several ways. Both methods are statistically based. Both are designed to minimize the sampling burden while improving the effectiveness of sampling, and both involve a preselection of employees prior to sampling to provide some assurance that operations are sampled in which employee exposures may be a problem. 
The zoning method of industrial hygiene sampling. The zoning method is used to determine the exposure for a group of employees based on their job similarity and the similarity of the environment in which they work. With zoning, the exposures for all people in a zone can be estimated, within some variation, from the exposures of the sampled group. According to $\mathrm{NIOSH}$, the average exposure obtained by sampling a subgroup of employees should be assigned to all employees because of considerable variation in employee exposure, even between employees doing the same job. There may be some difficulties in meeting the necessary requirements of the zoning method, particularly the requirement of "identifiability." To meet this requirement, a no-name classification such as occupational title and department must be used to assign employees to zones. The occupational titles must be well defined with regard to job responsibilities such that one occupational title group does not cross zone boundaries for a particular chemical agent. For example, the employees with the occupational title of senior machine operator in a given department are not necessarily exposed to the same average concentration of oil mist within some reasonable amount of variation. Several zones may be identified for similarity in job and environment, and the senior machinist occupational title could be found in each zone so that "identifiability" of employees in a zone by a no-name classification would be difficult. As a comparison, in a union plant, occupational titles are usually very defined in regard to actual work performed so that the zoning method could be used more readily. It can be assumed from general statistical considerations that the sample size becomes an increasing proportion of the population as the population size gets smaller. Where department size is small, zoning would provide little advantage over sampling all the operators in a department because the size of the sample approaches the size of the department. Zoning on an area or building-wide basis would provide increased effectiveness for a given number of samples provided zones could be adequately defined. Zoning on an area or buildingwide basis may involve difficulties in reporting employee results to an individual department manager in that he will need to understand why only one or two measurements were taken in the department when more than one or two employees are exposed. In addition, if the filing system for sampling records is kept on a departmental basis, the system may have to be changed to accommodate zone surveys.

The NIOSH method of industrial hygiene sampling. The object of the NIOSH method is to determine compliance with a standard. A decision is made for each personal sample after its upper confidence limit is calculated as to whether it is an overexposure, a possible overexposure, or within compliance. The upper confidence limit is compared to the standard or recommended exposure level and the action level, and the frequency or need for resampling is determined. The NIOSH method to determine compliance is currently being used by OSHA to evaluate samples obtained during inspections. The employee exposure determination and measurement strategy are included within the monitoring requirements in some OSHA-proposed regulations. The most difficult portion of the NIOSH method is to determine accurate coefficients of variation for the sampling and analytical methods. The total coefficients of variation are provided for many of the NIOSH procedures, but it is recommended that coefficients of variation be calculated by the individual industrial hygiene departments for their actual sampling and analytical procedures. NIOSH coefficients of variation can be used only when adequate maintenance and calibration facilities for sampling equipment and a quality control program for the analytical laboratory are used. Some NIOSH methods do not provide coefficients of variation, which therefore need to be calculated.

The two methods are not exclusive of one another. The zoning method can easily be used within the NIOSH method when no maximum risk employees can be identified for an operation. Methods for setting sampling priorities, such as one based on toxicity and exposure hazard potential, could also be incorporated into the general NIOSH method. Using the zoning method exclusively may not always be possible for all sampling, whereas the NIOSH method is applicable to most sampling. Another aspect to consider is that proposed OSHA regulations require the employer to identify all employees who may be exposed at or above the action level. The NIOSH method does this more readily while the zoning method indicates that a number of employees are exposed above the action level but does not identify them.

MSHA method of respirable dust sampling. The MSHA scheme of five samples collected by designated area or occupation to determine compliance with a permissible exposure limit is scientifically indefensible, as is the OSHA single sample (8-h timeweighted average) for compliance. Both sampling strategies are "guesses" at true conditions. Neither results in collection of meaningful long-term data for exposure or trends.

\section{Further implications of current regulatory and nonregulatory sampling strategies}

\section{Incentives for sampling}

A recent analysis of the OSHA enforcement strategy (9) concludes that the regulatory strategy, eg, NIOSH strategy, discourages employer exposure monitoring. In other words, increased monitoring activity in- 
creases the probability of detecting concentrations at the upper end of the concentration distribution. The employer must choose between sampling to detect hazard or sampling to confirm compliance. With the trend towards voluntary compliance in the US, it is predictable that sampling activity will be optimized to demonstrate compliance. Because only personal samples carry compliance implications in the US, another way to resolve this dilemma is to minimize personal samples while increasing area samples to detect hazard.

The suggested change of enforcement strategy is that, rather than a permissible exposure limit, a permissible distribution of concentrations be recognized by the regulators. This change would be incorporated into standards for ambient air quality where so many excursions per year are permitted above a certain contaminant concentration. This approach recognizes the real world nature of contaminant distribution and accommodates to it. Such an approach would require a major change in US rules.

\section{Relationship to biological exposure indices}

In 1984-1985, for the first time, the American Conference of Governmental Industrial Hygienists (ACGIH) included biological exposure indices in its threshold limit value publication (1). As stated by the ACGIH

\begin{abstract}
Biological exposure indices (BEIs) represent warning levels of biological response to the chemical, or warning levels of the chemical or its metabolic product(s), regardless of whether the chemical was inhaled, ingested, or absorbed via skin. Introduction of the BEI is a step in the evolution of the concept of TLVs. The BEI provides health personnel with an additional tool to provide protection for the worker .... The TLVs are a measure of the composition of the external environment surrounding the worker. BEIs are a measure of the amount of chemical absorbed into the body [p 59].
\end{abstract}

Biological exposure indices for carbon monoxide, ethyl benzene, styrene, toluene, trichloroethylene, and xylene have been presented by the ACGIH. Other such indices are undergoing review. Biological exposure indices are a major advance and should be part of an air sampling strategy, if possible. Measurement of the concentration of substances in breathing zone air does not assure that the worker is totally protected from adverse health effects from chemicals in the workplace. The indices reflect all routes of exposure; they are an adjunct tool to air sampling. If substituted for air sampling, it is a measurement of a possible damage function. Thus, air sampling leads to the belief that all exposures are acceptable; biological monitoring confirms it.

Another reason for using biological exposure indices as a second line of protection is the possibility that the monitored chemical may interact with other environment and workplace chemicals and this occurrence may stimulate or inhibit its metabolism and elimination, its toxicity in the worker thus being influenced.

Permit me to emphasize again that the appropriate role of biological monitoring is as a complement to environmental monitoring. Both techniques are valuable; each offers independem data that can help to assess exposure and potential effects.

In summary, defensible air sampling strategies exist and each surveillance program should be guided by such a strategy. There is little excuse for not utilizing a strategy, particularly when working in proximity to recommended standards and guidelines.

\section{References}

1. American Conference of Governmental Industrial Hygienists. Threshold limit values for chemical substances and physical agents in the work environment and biological exposure indices with intended changes for 1984-85. Cincinnati, OH 1984.

2. Corn M. Strategies of air sampling. In: McDonald JC, ed. Recent advances in occupational health. Churchill Livingstone, London 1981, pp 199-209.

3. Corn M, Esmen NA. Field sampling of airborne particulates. Am Laboratory July (1978).

4. Corn M, Esmen NA. Workplace exposure zones for classification of employee exposures to physical and chemical agents. Am Ind Assoc J 40 (1979) 47-57.

5. Corn M, Breysee P, Hall T, Chen G, Resby T, Swift DL. A critique of MSHA procedures for determination of permissible respirable coal mine dust containing free silica. Am Ind Hyg Assoc J 46 (1985) 4-8.

6. Esmen NA, Hammad YY. Log-normality of environmental sampling data. Environ Sci Health A12 (1971) 29-41.

7. Leidel N, Busch K, Lunch J. Occupational exposure sampling strategy. National Institute for Occupational Safety and Health, Cincinnati, OH 1977. (DHEW (NIOSH) publication number 77-173).

8. Ogden TLL, Birkett JL. The human head as a dust sampler. In: Walton WH, ed. Inhaled particles IV. Pergamon Press, Oxford 1977.

9. Rappaport SM. The risks of the game: An analysis of OSHA's enforcement strategy. Am J Ind Med 6 (1984) 291-303.

10. Wood JD, Burkett JL. External airflow effects on personal sampling. Ann Occup Hyg 22 (1979) 299310. 